this area? Delivering opioids via pumps that provide continuous infusions or fentanyl patches might overcome some of these problems. The dose would have to be titrated gradually, and a suitable placebo would be necessary. In trials comparing opioids in pill form with active placebo, perhaps the active placebo should contain one ingredient that gives nociceptive relief (e.g., NSAIDs) and a second ingredient that produces a calming but euphoric effect (e.g., a benzodiazepine such as lorazepam).

In traditional trials comparing oral opioids with placebo or NSAIDs, objective and detailed assessments of level of functioning and overall quality of life are better primary outcomes than pain. Participants' functioning, quality of life and pain should also be measured at a single point in time rather than being compared with baseline values.

\section{Tushar Mehta}

Substance Use Medical Service

St. Joseph's Health Centre

Toronto, Ont.

\section{REFERENCE}

I. Furlan AD, Sandoval JA, Mailis-Gagnon A, et al. Opioids for chronic noncancer pain: a metaanalysis of effectiveness and side effects. $C M A J$ 2006;I74(II):I589-94.

DOI:I0.I503/cmaj.ro6oI40

\section{[Three of the authors respond:]}

We thank Tushar Mehta for his comments regarding clinical trials to assess the efficacy of opioids for noncancer pain. Retrospective studies are indeed subject to recall bias, but we used only prospective controlled clinical trials in our meta-analysis. ${ }^{1}$ We extracted 2 outcome measures for our analyses: present pain intensity and present functional status. We avoided using outcomes measured as "better, the same or worse" because they are subject to recall bias. We were also careful to analyze the included studies with regard to valid outcome measures. The studies included in this review were judged to have valid outcome measures.

Mehta expresses doubts about the ability of a patient who is receiving opioids to validly report pain relief, but many studies have now established the validity of measures of pain relief as well as measures of functional status. Both of these types of outcome measures must be included if a trial is to be clinically relevant, and a valid prospective study must include comparisons of measurements taken at baseline and at subsequent points during treatment and follow-up in order to assess a therapy's efficacy.

In cases of substance abuse, opioids can be euphorigenic and continued use can lead to tolerance and even intermittent withdrawal symptoms. Our experience in pain relief clinics is that the great majority of people requiring opioids for pain relief are not addicted. Although it is normal for people to develop tolerance to opioids, nonaddicted patients with chronic noncancer pain do not commonly experience withdrawal phenomena if opioids are prescribed appropriately, and if sustainedrelease preparations are used.

\section{Andrea D. Furlan \\ Angela Mailis-Gagnon \\ Comprehensive Pain Program \\ Toronto Western Hospital \\ Toronto, Ont. \\ Eldon Tunks \\ Chedoke Rehabilitation Centre \\ Hamilton Health Sciences \\ Hamilton, Ont.}

\section{REFERENCE}

I. Furlan AD, Sandoval JA, Mailis-Gagnon A, et al. Opioids for chronic noncancer pain: a metaanalysis of effectiveness and side effects. $C M A J$ 2006;I74(II):I589-94.

DOI:I0.I503/cmaj.1070003

\section{Public involvement in guide-}

\section{line development}

Allan Detsky's timely editorial ${ }^{1}$ invites us to take a broader look at the biases that shape the recommendations in clinical practice guidelines, beyond financial links with the pharmaceutical industry. We strongly support Detsky's suggestion that nonexperts be included in guideline development panels, but we suggest that the value of public involvement should be judged not only on the basis of its impact on bias, but also in terms of democratic legitimacy and concordance with society's and patients' values.

Crafting recommendations involves making value judgments about the relative importance of competing goals and interests: maximizing health benefits for individuals and the population, promoting an equitable and effective allocation of resources, and respecting patients' autonomy. ${ }^{2}$ The crucial challenge is to ensure not only that recommendations are informed by the best available evidence, but also that the process is seen as transparent and legitimate. The quality of recommendations should be judged on the basis of their concordance with both the available evidence and the values of patients and society.

Patients are experts in their illness experience, life context and preferences. ${ }^{3}$ Like other experts, they have interests and are vulnerable to manipulation by the pharmaceutical industry. ${ }^{4}$ However, patient participation in guidelines committees could enhance the role of patients in clinical decision-making. ${ }^{5}$ Despite claims that they promote patient autonomy, clinical practice guidelines are rarely designed as instruments that facilitate a patient's choice.

Even if involvement by members of the general public in guideline development does not eliminate bias, at least their participation might bring a greater degree of legitimacy and accountability to the development process. Their participation would be in line with patients' desire to play a greater role in clinical decision-making.

\section{Antoine Boivin}

London School of Hygiene and

Tropical Medicine

London, UK

France Légaré

Associate Professor

Department of Family Medicine

Université Laval

Québec, Que.

\section{REFERENCES}

I. Detsky AS. Sources of bias for authors of clinical practice guidelines [editorial]. CMAJ 2006;175(9): I033.

2. Cohen J. Are clinical practice guidelines impartial? Int J Technol Assess Health Care 2004;20(4):415-20. 